\title{
Tip-Enhanced Infrared Imaging with Sub-10 nm Resolution and Hypersensitivity
}

Jian $\mathrm{Li}^{1}$, Junghoon Jahng ${ }^{2}$, Jie Pang ${ }^{1}$, William Morrison ${ }^{1}$, Jin $\mathrm{Li}^{1}$, Eun Seong Lee ${ }^{2}$, Jing-Juan $\mathrm{Xu}^{1}$, Hong-Yuan Chen ${ }^{1}$, Xing-Hua Xia ${ }^{1}$.

1. State Key lab of Analytical Chemistry for Life Science, School of Chemistry and Chemical Engineering, Nanjing University, 210023 Nanjing, China

2. Center for nanocharacterization, Korea Research Institute of Standards and Science, Daejeon 34113, Republic of Korea

3. Molecular Vista Inc., 6840 Via Del Oro, Suite 110, San Jose, CA 95119, USA. Corresponding author: X.H. Xia (xhxia@nju.edu.cn)

\section{Contents}

S1. Supplementary methods

S2. Simulations for the electromagnetic field distribution

S3. Calculations for the tip-enhanced thermal expansion and van der Waals force

S4. PiFM of MBA layer on different substrates

S5. AFM of Au film for imaging

S6. Hot spot simulation on Comsol

S7. PiFM and FTIR of BSA 


\section{S1. Supplementary methods}

\section{S1.1 Reagents}

$\mathrm{SiO}_{2} /$ Silicon wafer $\left(\mathrm{SiO}_{2}\right.$ thickness: $500 \mathrm{~nm}$; resistance of $\left.\mathrm{Si}: 1-15 \Omega \cdot \mathrm{cm}\right)$ was bought from Suzhou Crystal Silicon Electronic \& Tehchnology Co., Ltd. 16-Mercaptohexadecanoic acid (MDHA), 4-mercaptobenzoic acid(MBA) were purchased from Sigma-Aldrich. Bovine Serum Albumin (BSA) was purchased from Southern Biotechnology Associates, Inc., P. R. China. Other reagents and chemicals were of analytical grade. All reagents were used as received without further purification. MDHA and MBA solutions were prepared with ethanol and other solutions were prepared with Milli-Q water from a Millipore system.

\section{S1.2 Instruments}

AFM and PiFM were recorded on a VistaScope system (Molecular Vista, USA), and FTIR was collected on a Nicolet IS50 (Nicolet, USA). Vacuum evaporation of Au and Ag was carried out on a PVD75 Proline SP (Kurt, J Lesker, USA). Vacuum sputtering of Pt was carried out on a JEC-3000FC (JEOL, JP).

\section{S1.3 Preparation of the metal films}

Substrate was placed in a deposition cabin with the $\mathrm{SiO}_{2}$ side facing the metal target. After tuning parameters, the desired thickness of metal film was achieved. After deposition, the metal film was washed with deionized water and dried by $\mathrm{N}_{2}$.

\section{S1.4 Preparation of the samples}

For SAMs: Metal films were immersed in $10 \mathrm{mM}$ MDHA and MBA solution respectively for 24 h. After modification, the SAMs were washed with ethanol and dried by $\mathrm{N}_{2}$ sweeping. For the BSA layer, the gold film was dipped in $100 \mathrm{ng} / \mathrm{mL}$ BSA solution for $5 \mathrm{~s}$ and washed with deionized water following by drying with $\mathrm{N}_{2}$ sweeping.

\section{S1.5 Measurement of PiFM}

PiFM measurements were taken on a VistaScope microscope that is coupled to a QCL laser system from Block Engineering with a wavenumber resolution of $1 \mathrm{~cm}^{-1}$ and a tuning range from 770 to $1885 \mathrm{~cm}^{-1}$. The IR beam is focused on the sample with an angle of 30 degrees using a parabolic mirror, has a pulse duration of $40 \mathrm{~ns}$ and pulse energy of $0.1 \mu \mathrm{J}-2 \mu \mathrm{J}$ with incident wavelengths. The set point is set as $75 \%$ with an oscillation amplitude of $\sim 1 \mathrm{~nm}$. The microscope is operated in dynamic mode with $\mathrm{NCH}-\mathrm{Au} 300 \mathrm{kHz}$ non-contact cantilevers from Nanosensors. The cantilever was excited at its second resonance around $1.43 \mathrm{MHz}$. The measurements were carried out in an $\mathrm{N}_{2}$ environment to minimize the water background from environment. The collection time for each spectrum is around $1 \mathrm{~s}$, and the time per image is about 2 min with 1 line/s speed at $128 \times 128$ resolution. The spectrum is normalized with the background laser power profile spectrum.

\section{S1.6 IR characterization of molecules}

ATR-FTIR spectra of each molecule were recorded on a Nicolet IS 50. IR detection was carried out with a homemade ATR accessory and the diameter of the detection cell was $6 \mathrm{~mm}$. Unpolarized IR radiation was totally reflected at the $\mathrm{ZnSe}$ prism/solution interface with an incident angle $\theta=75^{\circ}$ and was detected with a liquid-nitrogen-cooled MCT detector. After adding 
the molecular solution into the accessory, the spectrum of each molecule was collected by taking the IR spectrum of ethanol (MDHA, MBA) or water (BSA) as the reference. For adsorption kinetics of BSA on Au film, a chemically prepared Au film was made on $\mathrm{ZnSe}$ prism as described in reference $\mathrm{S} 1$. The time-dependent ATR-surface enhanced infrared absorption spectra were recorded after the adding of $100 \mathrm{ng} / \mathrm{mL}$ BSA solution on prism surface by taking water spectrum as the reference. The spectral range was $4000-650 \mathrm{~cm}^{-1}$ with a resolution of 4 $\mathrm{cm}^{-1}$. 


\section{S2. Simulations for the electromagnetic field distribution}

According to the analytical theory of the field enhancement for the layered system ( $S 2)$, the field enhancement at the tip end is rigorously calculated with respect to the $\mathrm{SiO}_{2}$ substrate and a 90 $\mathrm{nm} \mathrm{Au}$ film on $\mathrm{SiO}_{2}$ as below. The simulation parameters are $\mathrm{R}=15 \mathrm{~nm}, \mathrm{~L}=300 \mathrm{~nm}, \mathrm{H}=1 \mathrm{~nm}$, and the incident electric field $\mathrm{E}_{0}$ is $3 \times 10^{5} \mathrm{~V} / \mathrm{m}$. 


\section{S3. Calculations for the tip-enhanced thermal expansion and the van der Waals force}

Calculating the absorbed power inside the monolayer by $P_{a b s}=\int a_{a b s} \frac{1}{2} c \varepsilon_{0}|E|^{2} d V$ with the absorption coefficient of $a_{a b s}=\frac{4 \pi}{\lambda} \frac{9 R e[n] \operatorname{Im}[n]}{\left(\operatorname{Re}[n]^{2}-\operatorname{Im}[n]^{2}\right)^{2}}$, one can estimate the thermal expansion of the monolayer. The $\sigma_{b a}, \rho_{b a}, C_{b a}$, and $\kappa_{b a}$ are $173 \times 10^{-6} \mathrm{~K}^{-1}, 1.5 \times 10^{3} \mathrm{~kg} / \mathrm{m}^{3}, 1202 \mathrm{~J} / \mathrm{kg} \cdot \mathrm{K}$ and $0.151 \mathrm{~W} / \mathrm{m} \cdot \mathrm{K}$, respectively, for the benzoic acid. According to the reference $S 3$, the maximum tip-enhanced thermal expansion can be estimated as:

$$
\Delta \mathrm{L}_{\max } \approx \sigma d \Delta \mathrm{T}_{\max } \approx \frac{\sigma \tau_{\mathrm{rel}}}{\rho C d} \int a_{a b s} \frac{1}{2} c \varepsilon_{0}|E|^{2} d z \text {. (S1) }
$$

where $\sigma$ is the linear thermal expansion coefficient and $\Delta \mathrm{T}_{\max }$ is given as $\Delta \mathrm{T}_{\max } \approx \frac{P_{\mathrm{abs}}}{\rho C V_{\text {heat }}} \tau_{\mathrm{rel}}$ for $\tau_{r e l}<\tau_{p}$ and $\Delta \mathrm{T}_{\max } \approx \frac{P_{\text {abs }}}{\rho C V_{\text {heat }}} \tau_{p}$ for $\tau_{r e l}>\tau_{p}(S 4)$. Because the thickness of the monolayer is around $1 \mathrm{~nm}$, by integrating the electric field inside the monolayer, then one can obtain the $\Delta \mathrm{L}$ of $\sim 5 \mathrm{pm}$ for the monolayer of benzoic acid at the vibrational resonance of $1694 \mathrm{~cm}^{-1}$, where the refractive index is 1.45 and the extinction coefficient is $0.87 \mathrm{~m}^{-1}$, resulted in the $a_{a b s}$ is $7.21 \times$ $10^{5} \mathrm{~m}^{-1}$. The modulated van der Waals force due to thermal expansion are given as

$$
\Delta \mathrm{F}_{\mathrm{vDW}} \approx-\frac{H_{\mathrm{eff}} R}{6} \frac{1}{H^{8}} \Delta L(\mathrm{~S} 2)
$$

where $H_{\text {eff, }} \mathrm{R}$ are the effective Hamaker constant and radius of the tip, respectively. The effective Hamaker constant between the Au tip and the benzoic acid is given as $H_{\text {eff }}=\sqrt{H_{\mathrm{Au}} \times H_{\mathrm{ba}}}=14$ $\times 10^{-20} \mathrm{~J}$ where $\mathrm{H}_{\text {Au }}$ is $45.3 \times 10^{-20} \mathrm{~J}$ and $\mathrm{H}_{\mathrm{ba}}$ is $4.3 \times 10^{-20} \mathrm{~J}$. Note that we implemented the Hamaker constant of the acetone or ethyl acetate as the $\mathrm{H}_{\mathrm{ba}}$, which has the similar van der Waals property (S13). Then, the modulated van der Waals force due to the thermal expansion for $\Delta \mathrm{L}=5$ $\mathrm{pm}$ is $\sim 1.6 \mathrm{pN}$ at $\mathrm{H}=1 \mathrm{~nm}$ gap distance. On the other hand, on the $\mathrm{SiO}_{2}$ substrate, the tipenhanced thermal expansion of the MBA is reduced by $\sim 0.2 \mathrm{pm}$ which corresponds to $\Delta \mathrm{F}_{\mathrm{vdW}} \sim$ $0.07 \mathrm{pN}$ at $\mathrm{H}=1 \mathrm{~nm}$ gap distance.

The $\sigma_{\text {MDHA }}, \rho_{\text {MDHA }}, C_{\text {MDHA }}$, and $\kappa_{\text {MDHA }}$ are $270 \times 10^{-6} \mathrm{~K}^{-1}, 0.942 \times 10^{3} \mathrm{~kg} / \mathrm{m}^{3}, 1831.9 \mathrm{~J} / \mathrm{kg} \cdot \mathrm{K}$ and $0.171 \mathrm{~W} / \mathrm{m} \cdot \mathrm{K}$, respectively, for the MDHA. The maximum tip-enhanced thermal expansion of MDHA whose thickness of the monolayer is around $2.5 \mathrm{~nm}$ can be estimated as $107 \mathrm{pm}$ on $\mathrm{Au}$ film, which corresponds to the $\Delta \mathrm{F}_{\mathrm{vdW}} \sim 13 \mathrm{pN}$. However, $\Delta \mathrm{L}$ of the MDHA is around $9 \mathrm{pm}$ which corresponds to $\Delta \mathrm{F}_{\mathrm{vdW}} \sim 0.8 \mathrm{pN}$. Thus, the PiFM signals of the monolayers are barely measurable on $\mathrm{SiO}_{2}$ substrate but they are well measurable on Au film, which corresponds to the experimental result.

All the parameters and where they are derived are listed in Table S1. 
Table S1. Details of the parameters used for PiFM calculation

\begin{tabular}{|c|c|c|c|c|}
\hline Parameters & Value & Adapted from & Value & Adapted from \\
\hline & \multicolumn{2}{|l|}{ For MBA } & \multicolumn{2}{|l|}{ For MDHA } \\
\hline $\begin{array}{l}\sigma(\text { Thermal } \\
\text { expansion) }\end{array}$ & $\begin{array}{l}173 \times 10^{-6} \\
\mathrm{~K}^{-1}\end{array}$ & $\begin{array}{l}\text { Volume } \\
\text { expansion } \\
\text { coefficient of } \\
\text { Benzoic } \\
\operatorname{acid}(S 5)\end{array}$ & $\begin{array}{l}270 \times 10^{-6} \\
\mathrm{~K}^{-1}\end{array}$ & $\begin{array}{l}\text { Volume expansion } \\
\text { coefficient of } \\
\text { Stearic } \operatorname{acid}(S 6)\end{array}$ \\
\hline C(Heat capacity) & $\begin{array}{l}1202 \\
\mathrm{~J} / \mathrm{kg} \cdot \mathrm{K}\end{array}$ & $\begin{array}{l}\text { Benzoic } \\
\operatorname{acid}(S 7)\end{array}$ & $\begin{array}{l}1831.9 \\
\mathrm{~J} / \mathrm{kg} \cdot \mathrm{K}\end{array}$ & Palmitic $\operatorname{acid}(S 8)$ \\
\hline $\begin{array}{l}\mathrm{k}_{\mathrm{ba}}(\text { Thermal } \\
\text { conductivity) }\end{array}$ & $\begin{array}{l}0.151 \\
\mathrm{~W} / \mathrm{m} \cdot \mathrm{K}\end{array}$ & $\begin{array}{l}\text { Benzoic acid } \\
\text { linear } \\
\text { fitting }(S 9)\end{array}$ & $\begin{array}{l}0.171 \\
\mathrm{~W} / \mathrm{m} \cdot \mathrm{K}\end{array}$ & $\begin{array}{l}\text { Myristic acid } \\
\text { linear fitting }(S 10)\end{array}$ \\
\hline $\begin{array}{l}\text { Refractive index at } \\
\text { vibrational } \\
\text { resonance }\end{array}$ & $\begin{array}{l}1.45+0.8 \\
7 \mathrm{i}(1690 \\
\mathrm{cm}-1)\end{array}$ & $\begin{array}{l}\text { Benzoic acid } \\
(S 11)\end{array}$ & $\begin{array}{l}1.43+0.24 \mathrm{i} \\
\left(1710 \mathrm{~cm}^{-1}\right)\end{array}$ & Oleic $\operatorname{acid}(S 12)$ \\
\hline $\begin{array}{l}\mathrm{H}_{\text {eff }} \text { effective } \\
\text { Hamaker constant }\end{array}$ & $\begin{array}{l}4.3 \times 10^{-} \\
{ }^{20} \mathrm{~J}\end{array}$ & $\begin{array}{l}\text { Benzoic acid } \\
(S 13)\end{array}$ & $6.7 \times 10^{-21} \mathrm{~J}$ & Palmitic $\operatorname{acid}(S 14)$ \\
\hline
\end{tabular}




\section{S4. PiFM of MBA layer on different substrates}

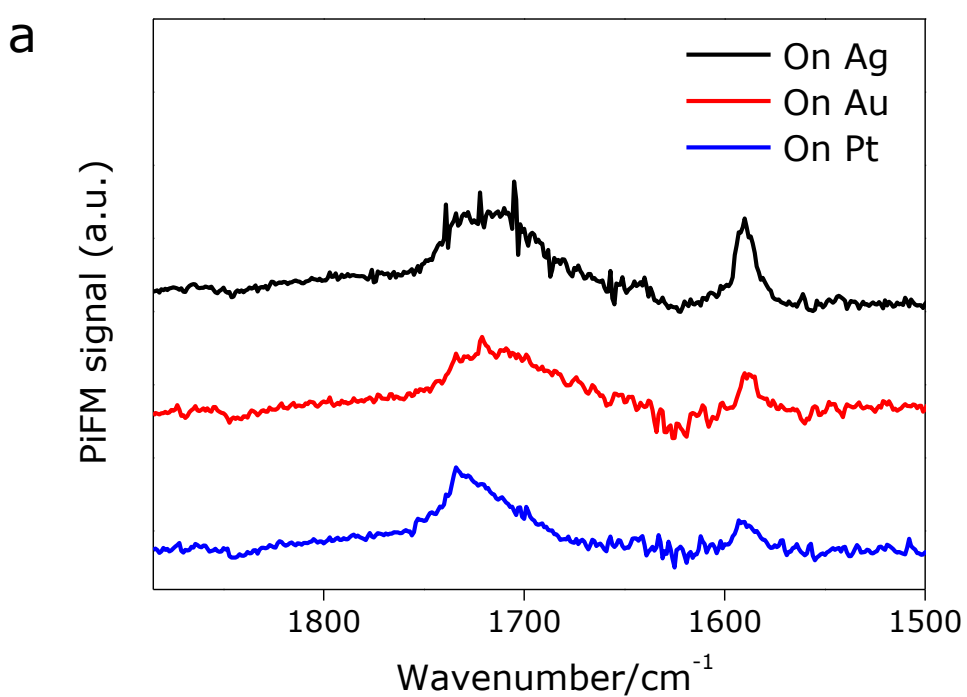

b

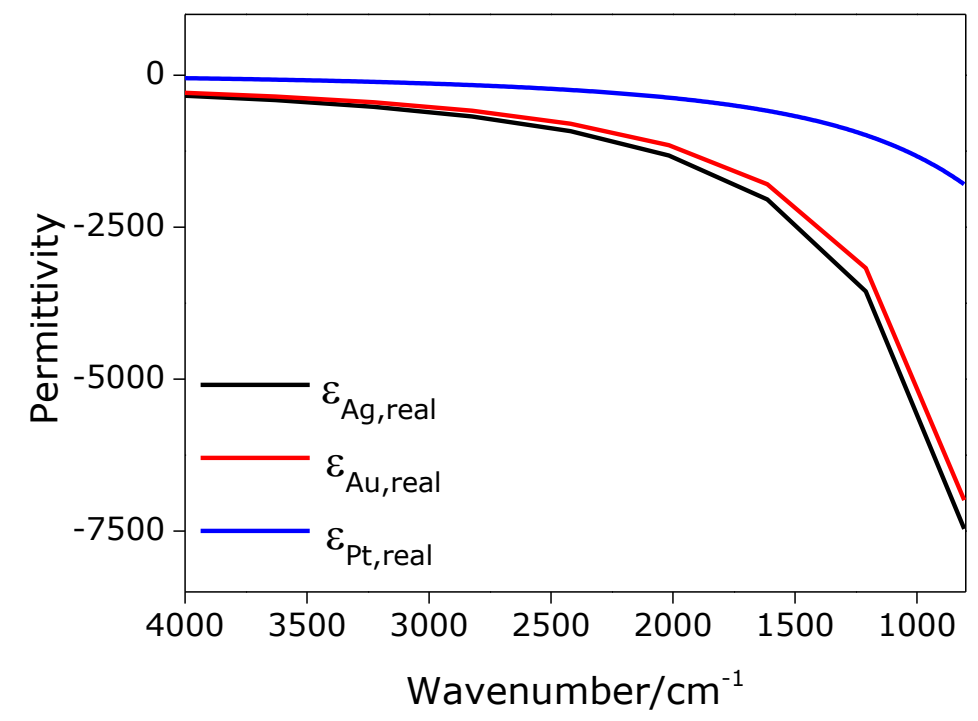

Figure S1. (a) PiFM spectra of MBA SAM on Ag, Au and Pt. (b) Permittivity real part plotting of $\mathrm{Ag}, \mathrm{Au}$ and $\mathrm{Pt}$ in the recorded region. It is clear that the three metals are highly reflective in the mid-IR region. The refractive index data are from $\operatorname{Ref}(S 15-S 16)$. 
S5. AFM of Au film for imaging

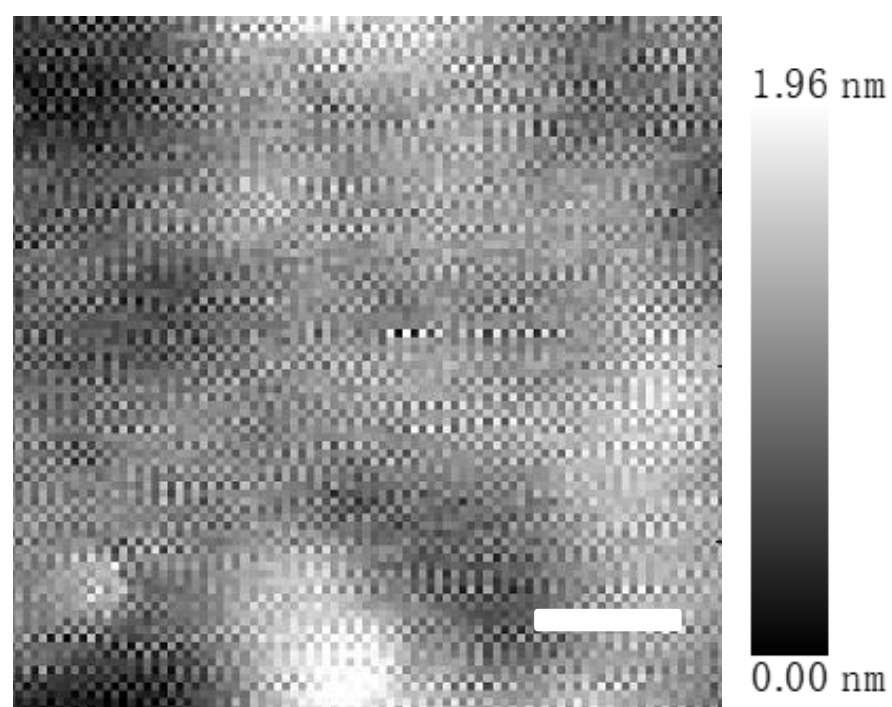

Figure S2. Topography of the gold. Scale bar: $100 \mathrm{~nm}$. 


\section{S6. Hot spot simulation on Comsol}

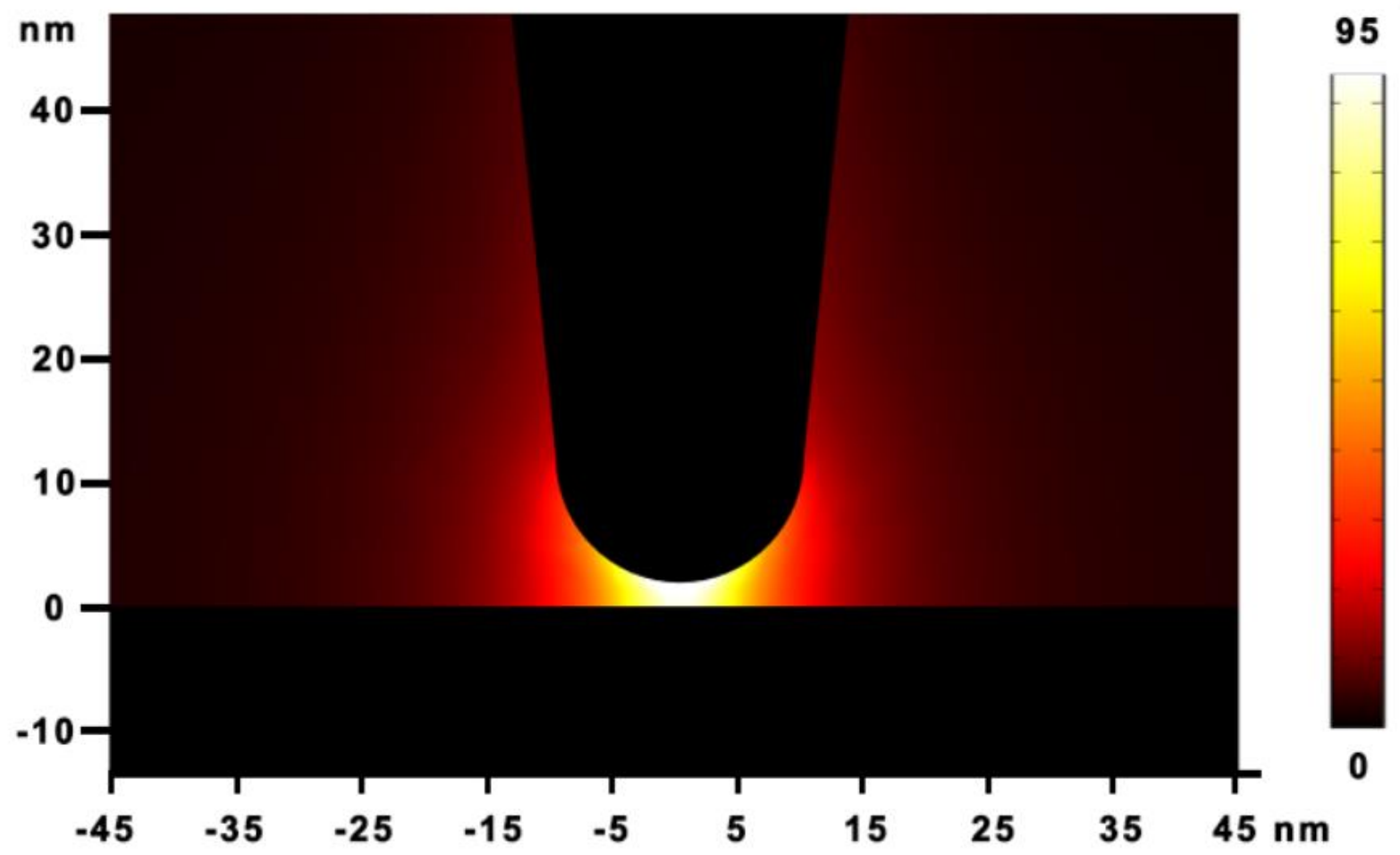

Figure S3. Field distribution of E field on the metal surface (xz plane). The hot spot was with around $5 \mathrm{~nm}$ diameter. Incident beam is set as p-polarized with 30 degree angle. The wavenumber is set as $1500 \mathrm{~cm}^{-1}$ as an arbitrary value while a whole IR region sweep does not show huge variation of the field distribution. The simulation is carried on comsol 3.5, scattering field is used to simulate the field distribution. The tip is set as a hemisphere with $10 \mathrm{~nm}$ radius head connected a cone with $30 \mathrm{~nm}$ end radius and $200 \mathrm{~nm}$ height. The substrate is set as $\mathrm{SiO}_{2} / \mathrm{Si}$ with a $100 \mathrm{~nm}$ thickness gold film as the experiment condition. 


\section{S7. PiFM and FTIR of BSA}
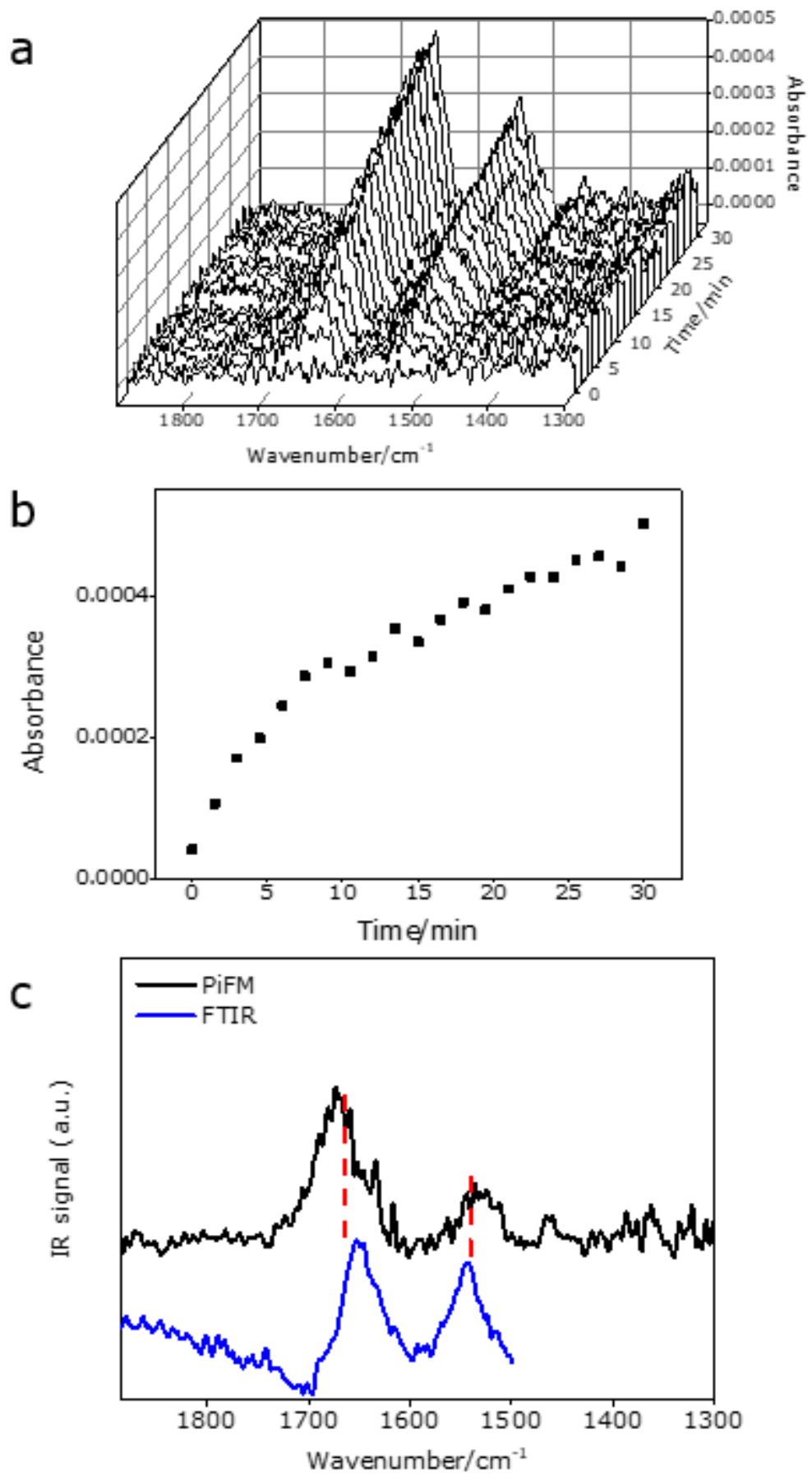

Figure S4. (a) Evolution of the antenna array enhanced ATR-SEIRAS spectra of $300 \mu \mathrm{L} 100$ $\mathrm{ng} / \mathrm{mL}$ BSA solution (b) Plot of the absorption intensity of amid I (black squares) from BSA as a function of adsorption time (c) PiFM (black curve) and ATR-FTIR (blue curve) spectra of BSA on gold and in solution, respectively. 
[S1] Bao, W. J.; Li, J.; Li, J.; Zhang, Q. W.; Liu, Y.; Shi, C. F.; Xia, X. H. Au/ZnSe-based surface enhanced infrared absorption spectroscopy as a universal platform for bioanalysis. Anal. Chem. 2018, 90, 3842-3848.

[S2] Hauer, B.; Engelhardt, A. P.; Taubner, T. Quasi-analytical model for scattering infrared near-field microscopy on layered systems. Opt. Express 2012, 20, 13173-13188.

[S3] Jahng, J.; Potma, E. O.; Lee, E. S. Tip-enhanced thermal expansion force for nanoscale chemical imaging and spectroscopy in photo-induced force microscopy. Anal. Chem. 2018, 90, 11054-11061.

[S4] Dazzi, A.; Glotin, F.; Carminati, R. Theory of infrared nanospectroscopy by photothermal induced resonance. J. Appl. Phys. 2010, 107, 124519.

[S5] Smith, E. R. The determination of the coefficient of cubical expansion of solid benzoic acid by means of a gas-filled dilatometer. Bureau of Standards Journal of Research 1931, 382, 903905.

[S6] Engineering ToolBox, (2014). Solids - Volume Temperature Expansion Coefficients. [online] Retrieved from: https://www.engineeringtoolbox.com/volum-expansion-coefficientssolids-d_1894.html (19 th, November, 2018)

[S7] Furukawa, G. T.; McCoskey, R. E.; King, G. J. Calorimetric properties of benzoic acid from $0^{\circ}$ to $410^{\circ}$ K. Journal of Research of the National Bureau of Standards, 1951, 47, 2251.

[S8] Saeed, R. M. Thermal characterization of phase change materials for thermal energy storage. Masters Theses, Missouri University, 2016.

[S9] Sun, T.; Teja, A. S. Density, viscosity, and thermal conductivity of aqueous benzoic acid mixtures between $375 \mathrm{~K}$ and 465 K. J. Chem. Eng. Data. 2004, 49, 1843-1846.

[S10] Wang, X.; Sun, T.; Teja, A. S. Density, viscosity, and thermal conductivity of eight carboxylic acids from (290.3 to 473.4) K. J. Chem. Eng. Data. 2016, 61, 2651-2658.

[S11] Yamamoto, K. Akio, M. Complex refractive index determination of bulk materials from infrared reflection spectra. Appl. Spectrosc. 1995, 49, 639-644.

[S12] McGinty, S.; Kapala, M. K.; Niedziela, R. F. Mid-infrared complex refractive indices for oleic acid and optical properties of model oleic acid/water aerosols. Phys. Chem. Chem.

Phys. 2009, 11, 7998-8004.

[S13] Croucher, M. D.; Halr, M. L. Hamaker constants and the principle of corresponding states. J. Phys. Chem. 1977, 81, 17.

[S14] Eom, N.; Parsons, D. F.; Craig, V. S. Measurement of long range attractive forces between hydrophobic surfaces produced by vapor phase adsorption of palmitic acid. Soft. Matter. 2017, 13, 8910-8921.

[S15] Babar, S.; Weaver, J. S. Optical constants of Cu, Ag, and Au revisited. Appl. Opt. 2015, 54, 477-481.

[S16] Rakić, A. D.; Djurišić, A. B.; Elazar, J. M.; Majewski, M. L. Optical properties of metallic films for vertical-cavity optoelectronic devices. Appl. Opt. 1998, 37, 5271-5283. 